\title{
Estimating Formation Mechanisms and Degree Distributions in Mixed Attachment Networks
}

\author{
Jan A. Medina, Jorge Finke, Camilo Rocha \\ Department of Electrical Engineering and Computer Science \\ Pontificia Universidad Javeriana \\ Cali, Colombia.
}

\begin{abstract}
Our work introduces an approach for estimating the contribution of attachment mechanisms to the formation of growing networks. We present a generic model in which growth is driven by the continuous attachment of new nodes according to random and preferential linkage with a fixed probability. Past approaches apply likelihood analysis to estimate the probability of occurrence of each mechanism at a particular network instance, exploiting the concavity of the likelihood function at each point in time. However, the probability of connecting to existing nodes, and consequently the likelihood function itself, varies as networks grow. We establish conditions under which applying likelihood analysis guarantees the existence of a local maximum of the time-varying likelihood function and prove that an expectation maximization algorithm provides a convergent estimate. Furthermore, the in-degree distributions of the nodes in the growing networks are analytically characterized. Simulations show that, under the proposed conditions, expectation maximization and maximum-likelihood accurately estimate the actual contribution of each mechanism, and in-degree distributions converge to stationary distributions.
\end{abstract}

Keywords: Complex networks, Network model, Statistical inference.

\section{Introduction}

The aim of a wide range of network models is to provide a framework to understand how linkage mechanisms for establishing links give rise to particular topological properties, including degree distributions [49], clustering [33], average path lengths [1, and community partitions [26. There has been a continuous and significant effort directed at formalizing such mechanisms and their role in the formation of networks like the Internet, the world wide web, and co-authorship associations $[31|5| 30|8| 2|19| 12|23| 13|17| 16|4| 34$.

The work in 4 explains the emergence of power law degree distributions as an outcome of two mechanisms, the addition of new nodes to the network (a growth mechanism) and the preference of new nodes to connect to nodes with a high degree (an attachment mechanism). The two mechanisms combined yield a degree distribution that asymptotically follow a power law. If the growth 
mechanism is combined with a uniform random attachment mechanism, with no preference for nodes with a high degree, then the resulting network follows an exponential function [30. However, a recent study shows that from nearly 1000 empirical networks less than $5 \%$ exhibit pure power law or exponential relationships [7].

Empirical networks have in general a richer diversity in structure, which suggests that, as networks grow, multiple mechanisms of attachment contribute to the resulting degree distributions [35]36. Citation networks, for example, have degree distributions which obey neither exponential nor power law, but mixed distributions 27. Evidence for such degree distributions are also found in opinion networks 29], and protein-protein interaction networks [1422.

It has been observed that networks in diverse domains (e.g., biological processes and social interaction) can have similar underlying generative mechanisms that make them hard to distinguish based purely on network structure [15]. Therefore, there has been especial interest in finding connections between the structure of networks and their underlying formation mechanisms 32 25. A number of models have been introduced to explain how attachment mechanisms, in particular, give rise to distinct topological properties. An important step in developing these models entails the problem of assessing the plausibility of each mechanism. Previous research tries to estimate the contribution of a mechanism using maximum-likelihood methods. Such methods determine the optimal estimate that best describes the contribution of each mechanism based on the number of new edges established at some point in time 35|36. The work in 36. assigns an adjustable weight to each mechanism. The optimal estimate represents the set of weights that maximize the likelihood of all new edges. However, as the network grows, applying standard maximum-likelihood estimation does not produce a consistent estimate over time.

Our work focuses on understanding how to obtain a convergent estimate of the contribution of multiple mechanisms that influence the evolution of growing networks. In particular, it aims to provide an analytical framework to evaluate the contribution of two attachment mechanisms, namely, random and preferential attachment. We extend the method in [35] and [36] by presenting conditions to guarantee the existence of a realistic maximum-likelihood estimate. Moreover, an expectation maximization algorithm is applied to evaluate the contribution of these two attachment mechanisms in one simulated network and one empirical citation network.

The contributions of this paper are the following. First, we characterize the roots of the likelihood function regarding the network parameters (Lemmata 1 and 2). Second, we present conditions under which the likelihood function has a maximum (Theorem 1) and an algorithm to estimate the contribution of the two attachment mechanisms (preferential and random attachment). Third, we use a discrete-time approach to characterize the in-degree distribution as a function of the parameters and contribution of each mechanisms (Theorem 2). Fourth, we show that the dynamics of the in-degree distribution converges to a stationary distribution (Corollary 2). Finally, we verify that the estimate of the contribution 
of random and preferential attachment yields a theoretical in-degree distribution that resembles empirical distributions of citation networks.

The remainder of the paper is organized as follows. Section 2 introduces the mixed attachment model. Section 3 presents the proposed estimation approach. Section 4 overviews the estimation based on expectation-maximization algorithm. Section 5 characterizes the in-degree distribution of the model. Section 6 presents simulation results. Section 7 draws the concluding remarks and some future work.

\section{The Network Model}

The network model used in this paper is an extension of the network model in [30, which supports directed networks and includes a response mechanism. It consists of three main mechanisms, namely, growth, attachment, and response. By growth we mean that the number of nodes in the network increases by one at each time step. Attachment refers to the fact that new nodes tend to connect to existing nodes, while response refers to the fact that existing nodes tend to connect to new nodes. In mathematical terms, the network model is parametric in a probability $\alpha$ and natural numbers $m$ and $\hat{m}$ governing the attachment and response mechanisms. Internally, the attachment mechanism creates $m>$ 0 outgoing edges from the new node and is characterized as a Bernoulli trial with parameter $\alpha$, where $\alpha$ represents the probability of establishing a new edge by preferential attachment and $1-\alpha$ by random attachment. The response mechanism creates $\hat{m} \geq 0$ incoming edges from the existing nodes to the new node by random attachment.

A network is represented as a directed graph $G_{t}=\left(V_{t}, E_{t}\right)$ with nodes $V_{t}$ and edges $E_{t} \subseteq V_{t} \times V_{t}$. A pair $(u, v) \in E_{t}$ represents a directed edge from a source node $u$ to a target node $v$. The expressions $k_{t}(u)$ and $\hat{k}_{t}(u)$ denote, respectively, the $i n$ - and out-degree of node $u \in V_{t}$. Moreover, $n_{t}$ and $e_{t}$ denote the number of nodes and the number of edges in the network at time $t$, respectively (i.e., $n_{t}=\left|V_{t}\right|$ and $\left.e_{t}=\left|E_{t}\right|\right)$.

Definition 1. The algorithm used in the network model goes as follows:

1. Growth: starting from a seed network $G_{0}$, at each time step $t>0$, a new node is added with $m$ outgoing edges that link the new node to $m$ different nodes already present in the network and $\hat{m}$ incoming edges that link $\hat{m}$ different nodes already present in the network to the new node.

2. Attachment: when choosing the $m$ nodes to which the new node connects, we assume that the probability $\pi_{t}(v \mid \alpha)$ that the new node will be connected to node $v$ is given by

$$
\pi_{t}(v \mid \alpha)=\alpha \pi_{t}^{p a}(v)+(1-\alpha) \pi_{t}^{r a}(v)
$$

where the probability of establishing an outgoing edge from the new node to the existing node $v$ due to preferential attachment is given by

$$
\pi_{t}^{p a}(v)=\frac{k_{t-1}(v)}{e_{t-1}}
$$


and due to random attachment by

$$
\pi_{t}^{r a}(v)=\frac{1}{n_{t-1}} .
$$

3. Response: when choosing the $\hat{m}$ nodes that connect to the new node, we assume that the probability $\eta_{t}(v)$ that an existing node $v$ connects to the new node is given by

$$
\eta_{t}(v)=\frac{1}{n_{t-1}}
$$

For the growth and response processes to be well-defined, the algorithm in Definition 1 assumes that the seed network has at least $\max \{m, \hat{m}\}$ nodes. Although it is not required by the algorithm, it will be further assumed that the seed network does not have self-loops and each node has at least one incoming edge. In this way, the networks generated by the algorithm do not contain selfloops and each node has non-zero in-degree. As explained before, the attachment mechanism is characterized as a Bernoulli trial with parameter $\alpha$, representing the probability of establishing a new edge via preferential attachment. For preferential attachment, the probability of establishing a new edge depends on the in-degree of the target node, meaning that the probability for an existing node of becoming a target node is directly proportional to its in-degree. For random attachment, the probability of establishing an edge to a target node follows a discrete uniform distribution. The response mechanism selects from the set of existing nodes uniformly at random which nodes connect to the new node.

Figure 1 illustrates the evolution of a seed network $G_{0}=\left(V_{0}, E_{0}\right)$ at time steps $t=1,2,3$ in the network model with $m=2$ and $\hat{m}=1$ (for the sake of simplicity in the illustration, the probability $\alpha$ is omitted). The seed network $G_{0}$ has nodes $V_{0}=\{1,2,3\}$ and edges $E_{0}=\{(1,2),(2,1),(2,3),(3,1)\}$. At time $t=1$, the set of nodes grows by adding the new node 4 , and by creating the new edges $(4,2),(4,3),(2,4)(m=2$ outgoing edges by the attachment mechanism and $\hat{m}=1$ incoming edges by the response mechanism). At time $t=2$, the new node 5 establishes $m=2$ outgoing edges and $\hat{m}=1$ incoming edges, as it is the case for the new node 6 at time $t=3$.

\section{Maximum Likelihood Analysis}

Consider the problem of determining the values of the $\alpha, m$, and $\hat{m}$ parameters used in the process of generating a sequence of netwroks $\left(G_{t}\right)_{t \leq T}$ by the algorithm in Definition 1 (see Section 2), for some seed network $G_{0}$ and $T \in \mathbb{N}$. Inspection on such a sequence could be enough for establishing the values of $m$ and $\hat{m}$. However, determining the value of $\alpha$ is, in general, a tall order. The purpose of this section is two-fold. On the one hand, it argues that recently published maximum likelihood analysis approaches (e.g., in [36]) do not produce reasonable estimates in some cases. On the other hand, it presents a new approach to provide better estimates and sufficient conditions under which such an analysis is feasible. 


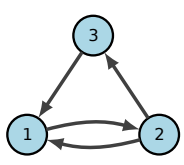

$\mathrm{G}_{0}$

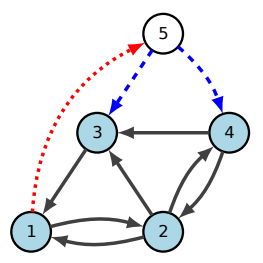

$\mathrm{G}_{2}$

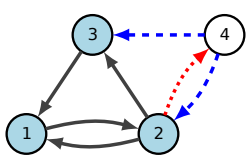

$\mathrm{G}_{1}$

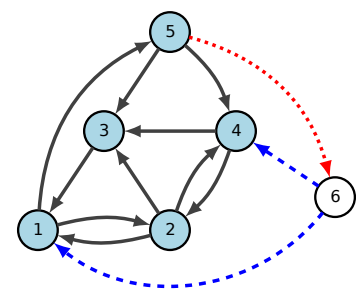

$\mathrm{G}_{3}$

Fig. 1: Network evolution from a given seed network $G_{0}$ in the network model. At each time step $t \geq 1$, a new node is added with $m=2$ outgoing edges (dashed line depicted in blue) and $\hat{m}=1$ incoming edges (dotted line depicted in red).

The likelihood of creating an edge from the new node $u$ at time $t$ to a node $v \in V_{t-1}$ is given by [36]:

$$
\begin{aligned}
\pi_{t}(v \mid \alpha) & =\alpha \pi_{t}^{p a}(v)+(1-\alpha) \pi_{t}^{r a}(v) \\
& =\alpha \frac{k_{t-1}(v)}{e_{t-1}}+(1-\alpha) \frac{1}{n_{t-1}} \\
& =\alpha\left(\frac{k_{t-1}(v)}{e_{t-1}}-\frac{1}{n_{t-1}}\right)+\frac{1}{n_{t-1}} .
\end{aligned}
$$

Since preferential attachment depends on the in-degree of the target node, Equation 5 can be written as a function of the in-degree of node $v$ with $k=k_{t-1}(v)$ :

$$
\pi_{t}(k \mid \alpha)=\alpha\left(\frac{k}{e_{t-1}}-\frac{1}{n_{t-1}}\right)+\frac{1}{n_{t-1}} .
$$

Definition 2. For $t \in \mathbb{N}$, let:

- $A_{t}$ be the multiset of in-degrees of nodes selected at time $t$ by the attachment mechanism in Definition 1 and

- $B_{t}=\left\{A_{1}, A_{2}, \ldots, A_{t}\right\}$ be the collection of all multisets of in-degrees of nodes selected up to time $t$.

At each time $t$, the family of sets $B_{t}$ is a random sample of size $t$. Since the elements of $B_{t}$ are independent and identically distributed (i.i.d.), it follows 
from Equation 6 that the likelihood function can be given by Equation 7

$$
\begin{aligned}
f_{t}(\alpha) & =\prod_{i=1}^{t} \prod_{j=1}^{\left|A_{i}\right|} \pi_{i}\left(k_{i j} \mid \alpha\right) \\
& =\prod_{i=1}^{t} \prod_{j=1}^{\left|A_{i}\right|}\left[\alpha\left(\frac{k_{i j}}{e_{i-1}}-\frac{1}{n_{i-1}}\right)+\frac{1}{n_{i-1}}\right],
\end{aligned}
$$

where $k_{i j}$ denotes the $j$-th element in $A_{i}$ (without loss of generality, the multiset $A_{i}$ is assumed sorted in ascending order). Note that $f_{t}(\alpha)$ is a polynomial in the indeterminate $\alpha$ and has order at most $m t$. If all $k \in A_{i}$ satisfy $k n_{i-1}-e_{i-1} \neq 0$ for $1 \leq i \leq t$, then the order of $f_{t}(\alpha)$, denoted $\operatorname{deg} f_{t}(\alpha)$, is $m t$.

Definition 3. For a network $G_{t}$, the function $f_{t}$ is called the $G_{t}$-likelihood function. The maximum likelihood estimator is defined as

$$
\hat{\alpha}=\underset{\alpha \in(0,1)}{\operatorname{argmax}} f_{t}(\alpha) .
$$

Consider the complete directed graph $G_{0}$ with 3 vertices, and a sequence $\left(G_{t}\right)_{t \leq 2000}$ generated by the algorithm in Definition 1 with parameters $m=5$, $\hat{m}=3$, and $\alpha=0.6$.

At each time $t$, likelihood analysis is applied to the multiset $A_{t}$ and to the collection $B_{t}$. Let the likelihood functions $f_{t}^{(1)}(\alpha)$ and $f_{t}^{(2)}(\alpha)$ be defined as:

$$
f_{t}^{(1)}(\alpha)=\prod_{k \in A_{t}} \pi_{t}(k \mid \alpha) \quad \text { and } \quad f_{t}^{(2)}(\alpha)=\prod_{i=1}^{t} \prod_{j=1}^{\left|A_{i}\right|} \pi_{i}\left(k_{i j} \mid \alpha\right) .
$$

At each time, an estimation of $\alpha$ is provided by each likelihood function. Figure 2 shows that the standard maximum-likelihood estimation with $f_{t}^{(1)}$ is not capable of producing a consistent estimate over time (this witnesses a counter-example to the approach in [36] for likelihood analysis). However, the proposed approach developed in the rest of this section, which supports the maximum-likelihood estimation with $f_{t}^{(2)}$, yields a better estimate. The remaining of this section establishes conditions under which the estimator based on the $G_{t}$-likelihood function produces a consistent estimate over time for the network model in Section 2 .

The next goal is to identify some key relationships between the number of nodes, the number of edges, and the number of roots of the $G_{t}$-likelihood function $f_{t}$, and then provide conditions under which it has a maximum.

Lemma 1. The degree of $f_{t}$ is $m$ t if and only if $k n_{i-1} \neq e_{i-1}$, for $1 \leq i \leq t$ and all $k \in A_{i}$. Moreover, for $k \in A_{i}$ such that $k n_{i-1} \neq e_{i-1}$ then

$$
\omega_{i k}=\frac{e_{i-1}}{e_{i-1}-k n_{i-1}},
$$

is a root of $f_{t}$. 

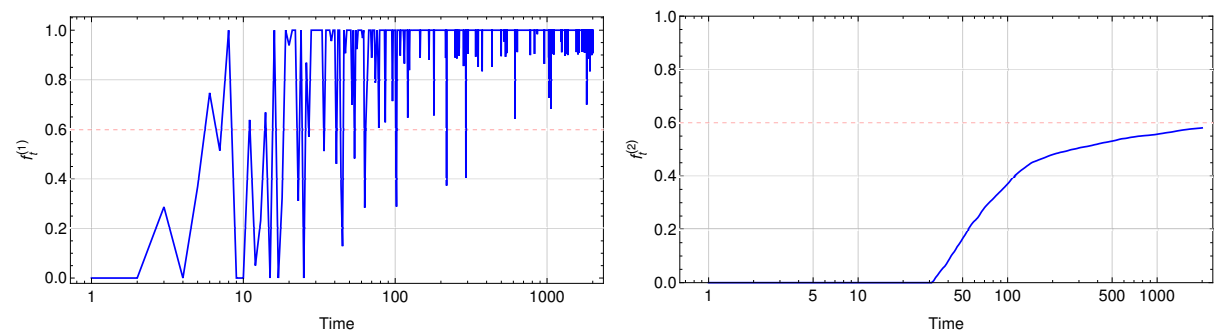

Fig. 2: Behavior of the parameter estimated by the likelihood functions $f_{t}^{(1)}$ and $f_{t}^{(2)}$, with $m=5, \hat{m}=3$, and $\alpha=0.6$.

Note that the root multiplicity of $\omega_{i j}$ corresponds to the number of times that the value $j$ appears in $A_{i}$.

Definition 4. Let $\Omega(p)$ be the real roots of a polynomial $p$ without repetitions, and $\Omega^{+}(p)$ and $\Omega^{-}(p)$ the set of positive and negative roots of $p$, respectively. The expression $\mu_{i}$ denotes the multiplicity of the root $\omega_{i}$ in $\Omega(p)$.

Recall from Section 2 that the seed network $G_{0}$ is assumed to have at least $m$ nodes, each with an in-degree of at least 1 (and without self-loops). This assumption is key for Lemma 2 to be applied. Otherwise, if there are nodes with in-degree 0 , then the minimum of the positive roots in $\Omega^{+}\left(f_{t}\right)$ would trivially be 1.

Lemma 2. If there is $A_{i} \in B_{t}$ and $k \in A_{i}$, with $0<k<\left\lfloor\frac{e_{i-1}}{n_{i-1}}\right\rfloor$, then $\Omega^{+}\left(f_{t}\right)$ and $\Omega^{-}\left(f_{t}\right)$ are non-empty sets. Moreover, $\min \Omega^{+}\left(f_{t}\right)=1+\frac{1}{m+\hat{m}-1}$ as $t \rightarrow \infty$.

Proof. Without loss of generality, assume that $\operatorname{deg} f_{t}=m t$. By Lemma 1) each root of $f_{t}$ can be written as

$$
\omega_{i k}=\frac{e_{i-1}}{e_{i-1}-k n_{i-1}} .
$$

Since $e_{i-1}>0$, the root $\omega_{k}$ is positive if and only if $e_{i-1}-k n_{i-1}>0$. This last claim is true for some $k \in A_{i}$ because $0<k<\left\lfloor\frac{e_{i-1}}{n_{i-1}}\right\rfloor$ by the hypothesis. Hence, $\Omega^{+}\left(f_{t}\right) \neq \emptyset$. Moreover, if $t$ is the greatest index for which $k=1$ belongs to $A_{t}$, then the denominator of Equation 8 yields the maximum positive integer. The minimum element of $\Omega^{+}\left(f_{t}\right)$ is then given by

$$
\begin{aligned}
\min \Omega^{+}\left(f_{t}\right) & =\frac{e_{t-1}}{e_{t-1}-n_{t-1}} \\
& =\frac{e_{t-1}-n_{t-1}+n_{t-1}}{e_{t-1}-n_{t-1}} \\
& =1+\frac{n_{t-1}}{e_{t-1}-n_{t-1}} .
\end{aligned}
$$


Furthermore, note that $e_{t}=e_{0}+(m+\hat{m}) t$ and $n_{t}=n_{0}+t$. Then

$$
\begin{aligned}
\lim _{t \rightarrow \infty}\left(\min \Omega^{+}\left(f_{t}\right)\right) & =\lim _{t \rightarrow \infty}\left(1+\frac{n_{t-1}}{e_{t-1}-n_{t-1}}\right) \\
& =\lim _{t \rightarrow \infty}\left(1+\frac{n_{0}+t-1}{e_{0}+(m+\hat{m})(t-1)-\left(n_{0}+t-1\right)}\right) \\
& =1+\frac{1}{m+\hat{m}-1} .
\end{aligned}
$$

Theorem 1. If $\Omega^{+}\left(f_{t}\right)$ and $\Omega^{-}\left(f_{t}\right)$ are non-empty, and the sum of the multiplicities of positive roots is even, then there exist $a \in \mathbb{R}^{-}, b \in \mathbb{R}^{+}$, and $\alpha \in \mathbb{R}$ such that $\alpha \in(a, b)$ is a local maximum of the $G_{t}$-likelihood function $f_{t}$.

Proof. Without loss of generality, assume that there is no $k \in A_{i}$ such that $k n_{i-1}=e_{i-1}$ for $1 \leq i \leq t$. Note that $f_{t}$ can be written as the following product

$$
\begin{aligned}
f_{t}(x) & =\prod_{i=1}^{t} \prod_{j=1}^{\left|A_{i}\right|} \frac{x\left(k n_{i-1}-e_{i-1}\right)+e_{i-1}}{e_{i-1} n_{i-1}} \\
& =\left[\prod_{i=1}^{t} \prod_{j=1}^{\left|A_{i}\right|} \frac{k n_{i-1}-e_{i-1}}{e_{i-1} n_{i-1}}\right]\left[\prod_{i=1}^{t} \prod_{j=1}^{\left|A_{i}\right|}\left(x-\frac{e_{i-1}}{e_{i-1}-k n_{i-1}}\right)\right] .
\end{aligned}
$$

Then, by letting $C=\prod_{i=1}^{t} \prod_{j=1}^{\left|A_{i}\right|} \frac{k n_{i-1}-e_{i-1}}{e_{i-1} n_{i-1}}$ and by Lemma 1 the following equation holds:

$$
f_{t}(\alpha)=C \prod_{i=1}^{t} \prod_{j=1}^{\left|A_{i}\right|}\left(x-\omega_{i k_{i j}}\right) .
$$

The hypothesis implies that the sets of real numbers $\Omega^{+}\left(f_{t}\right)$ and $\Omega^{-}\left(f_{t}\right)$ are finite and non-empty, and hence $\max \Omega^{-}\left(f_{t}\right)$ and $\min \Omega^{+}\left(f_{t}\right)$ exist. Let $a$ be the maximum element in $\Omega^{-}\left(f_{t}\right)$ and $b$ the minimum element in $\Omega^{+}\left(f_{t}\right)$. Note that the function $f_{t}$ is continuous and differentiable in $(a, b)$. Moreover, since $a$ and $b$ are roots of $f_{t}, f_{t}(a)=f_{t}(b)=0$. By Rolle's Theorem, there exists a constant $\alpha \in(a, b)$ such that $f^{\prime}(\alpha)=0$. That is, the maximum argument of $f_{t}$ exists for $\alpha \in(a, b)$.

To show that the point $\left(\alpha, f_{t}(\alpha)\right)$ is a local maximum of $f_{t}$, consider the following two cases.

Case 1. Assume that $\mu_{i}=1$ for each root $\omega_{i} \in \Omega\left(f_{t}\right)$. Since all the roots are simple, the function $f_{t}$ can be written as

$$
f_{t}(x)=C \prod_{\omega_{i} \in \Omega\left(f_{t}\right)}\left(x-\omega_{i}\right) .
$$


The derivative of $f_{t}$ with respect to $x$ is

$$
f^{\prime}(x)=C \sum_{\omega_{i} \in \Omega\left(f_{t}\right)} \prod_{\substack{\omega_{j} \in \Omega\left(f_{t}\right) \\ \omega_{j} \neq \omega_{i}}}\left(x-\omega_{j}\right) .
$$

Each term in the summation is a polynomial in the indeterminate $x$ and has degree $m t-1$. Let

$$
q_{i}(x)=\prod_{\substack{\omega_{j} \in \Omega\left(f_{t}\right) \\ \omega_{j} \neq \omega_{i}}}\left(x-\omega_{j}\right) .
$$

By the definition of $q_{i}$, the root $\omega_{i} \in \Omega\left(f_{t}\right)$ does not belong to $\Omega\left(q_{i}\right)$, i.e., $\Omega\left(q_{i}\right) \subsetneq \Omega\left(f_{t}\right)$. Based on Equation 10 . Equation 9 can be written as

$$
f^{\prime}(x)=C \sum_{\omega_{i} \in \Omega\left(f_{t}\right)} q_{i}(x) .
$$

By Lemma 1 there exists an in-degree $k \in A_{i}$ for some $A_{i} \in B_{t}$ satisfying $\omega_{i j}=a$. In particular, there exists $q_{s}$ such that $a$ does not belong to $\Omega\left(q_{s}\right)$. Therefore,

$$
f^{\prime}(a)=C q_{s}(a) .
$$

Since the first factor in Equation 12 is positive, the sign of $f^{\prime}(a)$ is the sign of $q_{s}(a)$. Note that the product in Equation 10 can be split into two factors, one containing the positive roots of $q_{s}$ and another one with the negative roots of $q_{s}$. More precisely,

$$
\begin{aligned}
q_{s}(x) & =\prod_{\substack{\omega_{j} \in \Omega\left(f_{t}\right) \\
\omega_{j} \neq a}}\left(x-\omega_{j}\right) \\
& =\prod_{\substack{\omega_{j} \in \Omega+\left(q_{s}\right) \\
\omega_{j} \neq a}}\left(x-\omega_{j}\right) \prod_{\substack{\omega_{j} \in \Omega-\left(q_{s}\right) \\
\omega_{j} \neq a}}\left(x-\omega_{j}\right) .
\end{aligned}
$$

The expression $q_{s}(a)$ can be rewritten as:

$$
q_{s}(a)=\prod_{\substack{\omega_{j} \in \Omega^{+}\left(q_{s}\right) \\ \omega_{j} \neq a}}\left(a-\omega_{j}\right) \prod_{\substack{\omega_{j} \in \Omega^{-}\left(q_{s}\right) \\ \omega_{j} \neq a}}\left(a-\omega_{j}\right) .
$$

In Equation 14, by the hypothesis, the first factor is a product of an even number of negative terms, which implies it is positive. The factors in the second product are positive because $a$ is the maximum element in $\Omega^{-}\left(f_{t}\right)$ and $a<-\omega_{i}$, for all $\omega_{i} \in \Omega^{-}\left(q_{s}\right)$.

By Lemma 1, there exists an in-degree $k^{\prime} \in A_{i^{\prime}}$ for some $A_{i^{\prime}} \in B_{t}$ such that $\omega_{i^{\prime} k^{\prime}}=b$; therefore, the sign of $f^{\prime}(b)$ depends on the sign of $q_{s^{\prime}}(b)$. Using the same argument as above:

$$
q_{s^{\prime}}(b)=\prod_{\substack{\omega_{j} \in \Omega^{+}\left(q_{s^{\prime}}\right) \\ \omega_{j} \neq b}}\left(b-\omega_{j}\right) \prod_{\substack{\omega_{j} \in \Omega^{-}\left(q_{s^{\prime}}\right) \\ \omega_{j} \neq b}}\left(b-\omega_{j}\right) .
$$


Note that $b-\omega_{j}<0$ because $b$ is the minimum positive root of $f_{t}$ and the roots are unique. Moreover, the number of terms in the first product in Equation 15 is an odd number. Thus, this product is negative. By Lemma 2 , all positive roots are greater than 1, so the second product in Equation 15 is positive. Consequently, $q_{s^{\prime}}(b)$ is negative. Therefore, $f^{\prime}(a)>0$ and $f^{\prime}(b)<0$. Hence, $(\alpha, f(\alpha))$ is a local maximum of $f_{t}$.

Case 2. Assume that there is at least one root $\omega_{i}$ in $\Omega\left(f_{t}\right)$ with $\mu_{i} \geq 2$. Since $\mu_{i} \geq 1$ for all roots $\omega_{i} \in \Omega\left(f_{t}\right)$, the function $f_{t}$ can be written as

$$
f_{t}(x)=C \prod_{\omega_{i} \in \Omega\left(f_{t}\right)}\left(x-\omega_{i}\right)^{\mu_{i}} .
$$

The derivative of $f_{t}$ with respect to $x$ is

$$
f^{\prime}(x)=C \sum_{\omega_{i} \in \Omega\left(f_{t}\right)}\left[\mu_{i}\left(x-\omega_{i}\right)^{\mu_{i}-1} \prod_{\substack{\omega_{j} \in \Omega\left(f_{t}\right) \\ \omega_{j} \neq \omega_{i}}}\left(x-\omega_{i}\right)^{\mu_{i}}\right] .
$$

The summands in Equation 16 are polynomials, each with the same roots as $f_{t}$, but with multiplicity of at most $\mu_{i}$. As for case 1 , the goal is to show that $f^{\prime}(a+\epsilon)>0$ and $f^{\prime}(b-\epsilon)<0$, for some $\epsilon>0$.

By defintion the elements of $\Omega\left(f_{t}\right)$ are unique, then it can be indexed as $\Omega\left(f_{t}\right)=\left\{\omega_{i_{1}}, \omega_{i_{2}}, \ldots, \omega_{i_{s}}\right\}$. Expanding the second factor in Equation 16

$$
\begin{aligned}
& \sum_{\omega_{i} \in \Omega\left(f_{t}\right)}\left[\mu_{i}\left(x-\omega_{i}\right)^{\mu_{i}-1} \prod_{\substack{\omega_{j} \in \Omega\left(f_{t}\right) \\
\omega_{j} \neq \omega_{i}}}\left(x-\omega_{i}\right)^{\mu_{i}}\right] \\
& =\mu_{i_{1}}\left(x-\omega_{i_{1}}\right)^{\mu_{i_{1}}-1}\left[\left(x-\omega_{i_{2}}\right)^{\mu_{i_{2}}}\left(x-\omega_{i_{3}}\right)^{\mu_{i_{3}}} \ldots\right] \\
& +\mu_{i_{2}}\left(x-\omega_{i_{2}}\right)^{\mu_{i_{2}}-1}\left[\left(x-\omega_{i_{1}}\right)^{\mu_{i_{1}}}\left(x-\omega_{i_{3}}\right)^{\mu_{i_{3}}} \ldots\right] \\
& +\mu_{i_{3}}\left(x-\omega_{i_{3}}\right)^{\mu_{i_{3}}-1}\left[\left(x-\omega_{i_{1}}\right)^{\mu_{i_{1}}}\left(x-\omega_{i_{2}}\right)^{\mu_{i_{2}}} \ldots\right] \\
& +\cdots \\
& =\left[\prod_{\omega_{i} \in \Omega\left(f_{t}\right)}\left(x-\omega_{i}\right)^{\mu_{i}-1}\right] \sum_{\omega_{i} \in \Omega\left(f_{t}\right)}\left[\mu_{i} \prod_{\substack{\omega_{j} \in \Omega\left(f_{t}\right) \\
\omega_{j} \neq \omega_{i}}}\left(x-\omega_{j}\right)\right] .
\end{aligned}
$$

Using Equation 18, Equation 16 can be written as

$$
f^{\prime}(x)=C\left[\prod_{\omega_{i} \in \Omega\left(f_{t}\right)}\left(x-\omega_{i}\right)^{\mu_{i}-1}\right] \sum_{\omega_{i} \in \Omega\left(f_{t}\right)}\left[\mu_{i} \prod_{\substack{\omega_{j} \in \Omega\left(f_{t}\right) \\ \omega_{j} \neq \omega_{i}}}\left(x-\omega_{j}\right)\right] .
$$

In Equation 19 the factor $\sum_{\omega_{i} \in \Omega\left(f_{t}\right)}\left[\mu_{k} \prod_{\substack{\omega_{j} \in \Omega\left(f_{t}\right) \\ \omega_{j} \neq \omega_{i}}}\left(x-\omega_{j}\right)\right]$ is equal to $\sum_{\omega_{i} \in \Omega\left(f_{t}\right)} \mu_{i} q_{i}(x)$.

On the one hand, $q_{i}(a)>0$ if and only if $\left|\Omega\left(f_{t}\right)\right|$ is even. Since $q_{i}$ is continuous 
over its domain, there exist $M>0$ and $\delta>0$ such that if $(a+\epsilon) \in(a-\epsilon, b+\epsilon)$ and $|(a+\epsilon)-a|<\delta$, then $q_{i}(a+\epsilon)>0[3$. The second term in Equation 19 can be rewritten as

$$
\prod_{\omega_{i} \in \Omega^{-}\left(f_{t}\right)}\left(x-\omega_{i}\right)^{\mu_{i}-1} \prod_{\omega_{i} \in \Omega^{+}\left(f_{t}\right)}\left(x-\omega_{i}\right)^{\mu_{i}-1} .
$$

The first term in Equation 20 is positive evaluated at $a+\epsilon$. The second term has the property that the sum of all exponents is an even number and, evaluated at $a+\epsilon$, is also positive. Hence, $f^{\prime}(a+\epsilon)>0$.

If $\left|\Omega\left(f_{t}\right)\right|$ is odd, the function $q_{i}$ evaluated at $a$ is negative. Using the same argument as above, it can be shown that $q_{i}(a+\epsilon)<0$. There exists an odd number of even multiplicities by the hypothesis. Consequently, the second factor in Equation 20 evaluated at $a+\epsilon$, is negative. In either case $f^{\prime}(a+\epsilon)>$ 0 . Similarly, it can be shown that $f^{\prime}(b-\epsilon)<0$.

Therefore, $(\alpha, f(\alpha))$ is a local maximum of $f_{t}$.

To illustrate the application of Theorem 1 , consider the complete graph $G_{0}$ with $N_{0}=3$ nodes, and a sequence $\left(G_{t}\right)_{t \leq 20000}$ generated by the algorithm in Definition 1 with parameters $m=5, \hat{m}=3$, and $\alpha=0.6$. At each time $t$, the $G_{t}$-likelihood function is the one in Definition 3 . An estimate for $\alpha$ is computed maximizing the $G_{t}$-likelihood function under the conditions of Theorem 11. Figure 3 shows how maximizing the $G_{t}$-likelihood function yields the estimate $\tilde{\alpha}$ for the parameter $\alpha$.

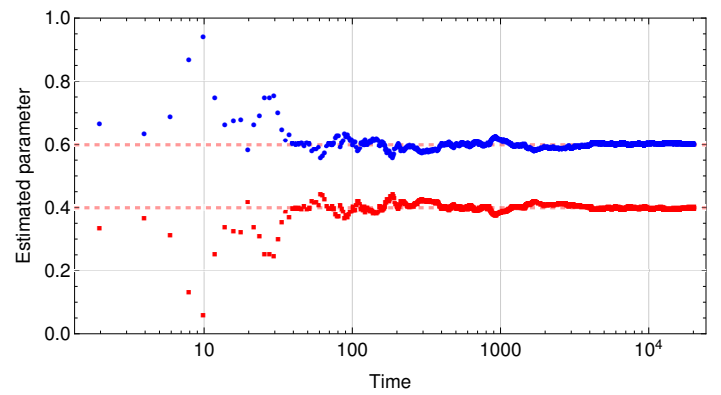

Fig. 3: Convergence of the parameters associated to preferential (dots) and random (filled squares) attachment mechanisms by application of Theorem 1 for the complete graph $G_{0}$ with $N_{0}=3$ nodes, and a sequence $\left(G_{t}\right)_{t \leq 20000}$ generated by the algorithm in Definition 1 with parameters $m=5, \hat{m}=3$, and $\alpha=0.6$.

\section{Using an Expectation-Maximization Algorithm}

This section presents how determining the contribution of the two attachment mechanisms used by the algorithm in Definition 1 is equivalent to computing 
the likelihood estimator with an Expectation-Maximization (EM) algorithm. The basic idea is to associate a complete-data problem, which is better suited for maximum likelihood estimation, to a given incomplete-data problem, for which the same estimation can become a wild-goose chase. For an EM algorithm to be effective, two important requirements need to be met: first, it needs to be proved convergent; second, it needs to be efficient. This section addresses these two requirements by identifying sufficient conditions for the algorithm to converge and by presenting a recursive definition for estimating $\alpha$, which can be used for incremental computation in the EM algorithm.

Definition 5. Let $X_{t}$ be a random variable that characterizes the in-degree of the selected nodes due to an attachment mechanism up to time $t$ from $G_{t}$. Moreover, let $\mathbb{P}(x \mid \theta)$ denote the probability mass function (pmf) as a function of the parameter vector $\theta$.

Recall that, for a fixed time $t$, Equation 7 defines the likelihood of forming an edge $(u, v)$ from the new node $u$ to node $v \in V_{t-1}$ through an attachment mechanism. Such an equation can be rewritten as:

$$
f_{t}(\alpha)=\prod_{i=1}^{t} \prod_{j=1}^{\left|A_{i}\right|}\left[\alpha \frac{k_{i j}}{e_{i-1}}+(1-\alpha) \frac{1}{n_{i-1}}\right] .
$$

The set $B_{t}=\left\{A_{1}, \ldots, A_{t}\right\}$ is a collection of samples of length $m$ generated by the random variable $X_{t}$ (see Definition 5).

The EM algorithm computes iteratively a maximum likelihood estimator for data with unobserved variables [10]6. In this case, the input to the EM algorithm comes from a mixed distribution in which the mixture weight is unknown. Based on Equation 21, the incomplete likelihood function can be modeled as follows:

$$
\mathcal{L}\left(\alpha \mid B_{t}\right)=\sum_{i=1}^{t} \sum_{j=1}^{\left|A_{i}\right|} \log \left(\alpha \frac{k_{i j}}{e_{i-1}}+(1-\alpha) \frac{1}{n_{i-1}}\right) .
$$

where $k_{i j}$ is the $j$-th in-degree in the sample $A_{i}$ (which, as assumed in Section 3 and without loss of generality, is sorted in ascending order) and $\left|A_{i}\right|$ is the length of $A_{i}$. The attachment mechanism operates on two groups of nodes. One group consists of nodes that attach to the network using preferential attachment and the other of the nodes that attach using random attachment. Consider a binary variable $Y$ for each occurrence of an in-degree $k$ to indicate whether the observation has been selected by preferential attachment or by random attachment. For $k_{i j}$, define $y_{i j}=1$ if $k_{i j}$ is in group one and $y_{i j}=0$ otherwise 24.

Notice that $\mathbb{P}\left(y_{i j}=1 \mid \alpha\right)=\alpha$ and $\mathbb{P}\left(y_{i j}=0 \mid \alpha\right)=1-\alpha$. The conditional probabilities of the data and the unobservable data are:

$$
\begin{aligned}
\mathbb{P}\left(k_{i j} \mid y_{i j}, \alpha\right) & =\left(\frac{k_{i j}}{e_{i-1}}\right)^{y_{i j}}\left(\frac{1}{n_{i-1}}\right)^{1-y_{i j}} \\
\mathbb{P}\left(y_{i j} \mid \alpha\right) & =\alpha^{y_{i j}}(1-\alpha)^{1-y_{i j}} .
\end{aligned}
$$


By defining $Z=\left(B_{t}, Y\right)$ as the complete-data, the complete log-likelihood function is given by

$$
\begin{aligned}
\mathcal{L}_{c}(Z \mid \alpha)= & \sum_{i=1}^{t} \sum_{j=1}^{\left|A_{i}\right|} \log \left(\mathbb{P}\left(k_{i j} \mid y_{i j}, \alpha\right) \mathbb{P}\left(y_{i j} \mid \alpha\right)\right. \\
= & \sum_{i=1}^{t} \sum_{j=1}^{\left|A_{i}\right|} \log \left(\left[\alpha \frac{k_{i j}}{e_{i-1}}\right]^{y_{i j}}\left[(1-\alpha) \frac{1}{n_{i-1}}\right]^{1-y_{i j}}\right) \\
= & \sum_{i=1}^{t} \sum_{j=1}^{\left|A_{i}\right|} y_{i j} \log \left(\alpha \frac{k_{i j}}{e_{i-1}}\right) \\
& +\sum_{i=1}^{t} \sum_{j=1}^{\left|A_{i}\right|}\left(1-y_{i j}\right) \log \left((1-\alpha) \frac{1}{n_{i-1}}\right)
\end{aligned}
$$

Equation 23 is linear in the unobservable data $y_{i j}$. Consider a function $Q\left(\alpha \mid \alpha^{(d)}\right)$ that represents the conditional expectation given the observed data using the $d$-th fit for the unknown parameter $\alpha$. In particular,

$$
\begin{aligned}
Q\left(\alpha \mid \alpha^{(d)}\right)= & \sum_{i=1}^{t} \sum_{j=1}^{\left|A_{i}\right|} \mathbb{P}\left(y_{i j}=1 \mid k_{i j}, \alpha^{(d)}\right) \log \left(\alpha \frac{k_{i j}}{e_{i-1}}\right) \\
& +\sum_{i=1}^{t} \sum_{j=1}^{\left|A_{i}\right|} \mathbb{P}\left(y_{i j}=0 \mid k_{i j}, \alpha^{(d)}\right) \log \left((1-\alpha) \frac{1}{n_{i-1}}\right) .
\end{aligned}
$$

By Bayes' Theorem,

$$
\begin{aligned}
\mathbb{P}\left(y_{i j}=1 \mid k_{i j}, \alpha^{(d)}\right) & =\frac{\mathbb{P}\left(k_{i j} \mid y_{i j}=1, \alpha^{(d)}\right) \mathbb{P}\left(y_{i j}=1 \mid \alpha^{(d)}\right)}{\mathbb{P}\left(k_{i} \mid \alpha^{(d)}\right)} \\
& =\frac{\frac{k_{i j}}{e_{i-1}} \alpha^{(d)}}{\frac{k_{i j}}{e_{i-1}} \alpha^{(d)}+\frac{1}{n_{i-1}}\left(1-\alpha^{(d)}\right)}
\end{aligned}
$$

And

$$
\begin{aligned}
\mathbb{P}\left(y_{i j}=0 \mid k_{i j}, \alpha^{(d)}\right) & =1-\mathbb{P}\left(y_{i j}=1 \mid k_{i j}, \alpha^{(d)}\right) \\
& =\frac{\frac{1}{e_{i-1}}\left(1-\alpha^{(d)}\right)}{\frac{k_{i j}}{e_{i-1}} \alpha^{(d)}+\frac{1}{n_{i-1}}\left(1-\alpha^{(d)}\right)}
\end{aligned}
$$

Consider $x_{1}=\alpha$ and $x_{2}=1-\alpha$. In order to maximize $Q\left(\alpha \mid \alpha^{(d)}\right)$, the Lagrange multiplier $\lambda$ with the constrain $x_{1}+x_{2}=1$ is introduced with the goal of solving the following equation:

$$
\sum_{i=1}^{t} \sum_{j=1}^{\left|A_{i}\right|} \frac{\mathbb{P}\left(k_{i j} \mid y_{i j}=1, \alpha^{(d)}\right)}{x_{1}}-\lambda=0
$$


Equation 27 has the solution:

$$
\alpha^{(d+1)}=\frac{1}{\sum_{i=1}^{t}\left|A_{i}\right|} \sum_{i=1}^{t} \sum_{j=1}^{\left|A_{i}\right|} \frac{\frac{k_{i j}}{e_{i-1}} \alpha^{(d)}}{\frac{k_{i j}}{e_{i-1}} \alpha^{(d)}+\frac{1}{n_{i-1}}\left(1-\alpha^{(d)}\right)} .
$$

Equation 28 provides a recursive formulation of $\alpha$ that can be used for estimation purposes in Algorithm 1, namely, in the EM algorithm. This algorithm computes an estimate for $\alpha$ on an input column block matrix $\left[A_{1} A_{2} \cdots A_{t}\right]^{T}$ where $A_{i} \in \mathbb{Z}^{m \times 3}$. For a fixed $A_{i}$, the values $a_{i 1}, a_{i 2}$, and $a_{i 3}$ denote the in-degree of the target node, the number of edges, and the number of nodes in the network, respectively. Line 3 computes the probability of the new edge forming by preferential attachment or random attachment mechanism (it implements the E-step of the EM algorithm). Lines 2-6 approximate the value for the parameter $\alpha$ until a sufficiently accurate value is reached. Note that the recursive definition of $\alpha$ presented in Equation 28 is used in line 4 (it implements the M-step of the EM algorithm). Finally, the convergence of Algorithm 1 is obtained as a corollary of Lemma 2.1 in [11].

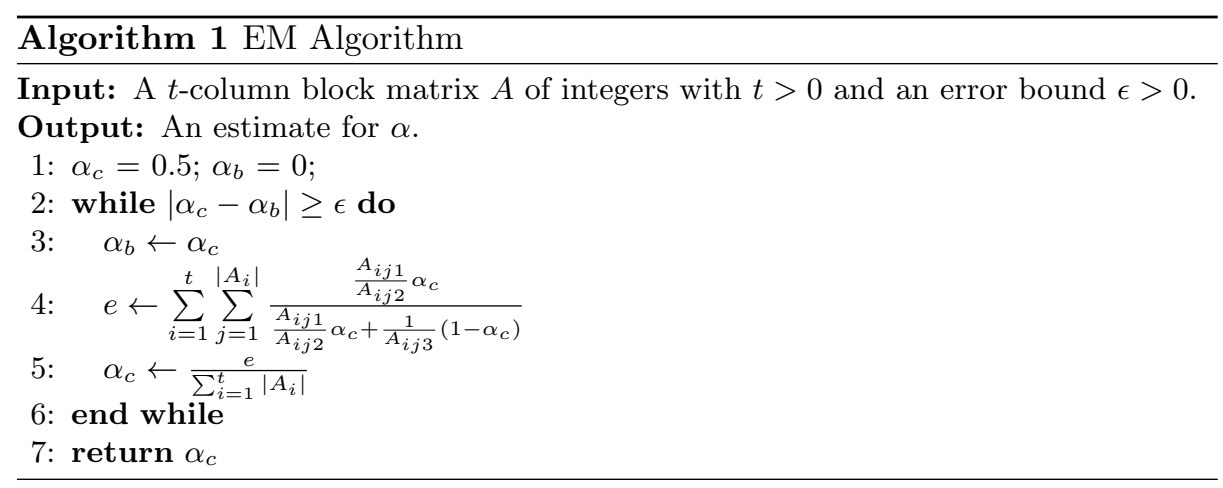

Corollary 1. Algorithm 1 converges.

The main observation in the proof of Corollary 1 is that the function $\log \mathbb{P}\left(\alpha \mid B_{t}\right)$ in Equation 22 is concave.

Recall the complete graph $G_{0}$ with $N_{0}=3$ nodes, and a sequence $\left(G_{t}\right)_{t \leq 20000}$ generated by the algorithm in Definition 1 with parameters $m=5, \hat{m}=3$, and $\alpha=0.6$. An estimate for $\alpha$ computed by Algorithm 1 (i.e., the EM Algorithm) is depicted in Figure 4. The final estimate computed for $\alpha$ by the EM Algorithm closely approximates the one computed by using Theorem 1 at the end of Section 3 


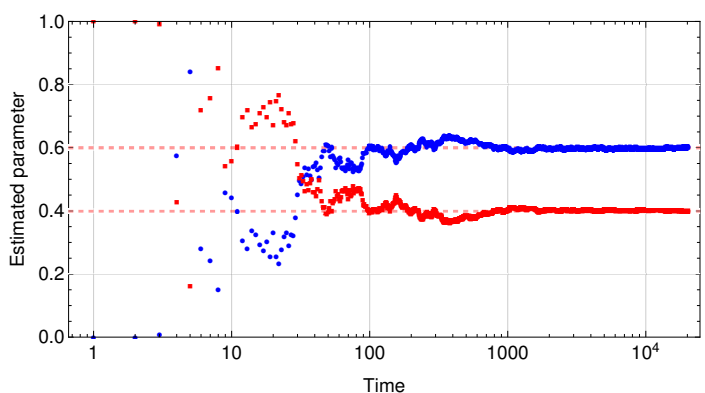

Fig. 4: Convergence of the parameters associated to preferential (dots) and random (filled squares) attachment mechanisms by the EM Algorithm for the complete graph $G_{0}$ with $N_{0}=3$ nodes, and a sequence $\left(G_{t}\right)_{t \leq 20000}$ generated by the algorithm in Definition 1 with parameters $m=5, \hat{m}=3$, and $\alpha=0.6$.

\section{In-degree Distribution}

This section characterizes the in-degree distribution of the nodes in a sequence of networks $\left(G_{t}\right)_{t \leq T}$ generated by the algorithm presented in Definition 1. for some $T \in \mathbb{N}$, from a given seed network $G_{0}$. It also presents how the dynamics of the in-degree distribution converges to a stationary distribution and illustrates the approach with experiments on sequences of networks $\left(G_{t}\right)_{t \leq T}$.

Definition 6. Let $K_{t}$ be a random variable that characterizes the in-degree of a node selected uniformly at random at time $t$ from $G_{t}$. Moreover, let $P_{t}(k)=$ $\mathbb{P}\left(K_{t}=k\right)$ denote the probability that $K_{t}$ is equal to $k$ at time $t$.

The notion of asymptotic equivalence between two real sequences is used to prove the existence of $\lim _{t \rightarrow \infty} P_{t}(k)$. Theorem 2 ensures that the probability of the in-degree distribution of the model converges.

Theorem 2. For $t \in \mathbb{N}, \lim _{t \rightarrow \infty} P_{t}(k)$ exists for all $k \geq \hat{m}$.

Proof. First, note that the expected number of nodes of in-degree $k \geq \hat{m}$ is

$$
\begin{aligned}
n_{t} P_{t}(k)= & n_{t-1} P_{t-1}(k)-m \pi_{t}(k \mid \alpha) n_{t-1} P_{t-1}(k) \\
& +m \pi_{t}(k-1 \mid \alpha) n_{t-1} P_{t-1}(k-1) .
\end{aligned}
$$

According to Equation 6

$$
\begin{aligned}
n_{t} P_{t}(k)= & \left(n_{t-1}-\frac{m \alpha k n_{t-1}}{e_{t-1}}-m(1-\alpha)\right) P_{t-1}(k) \\
& +\left(\frac{m \alpha(k-1) n_{t-1}}{e_{t-1}}+m(1-\alpha)\right) P_{t-1}(k-1) .
\end{aligned}
$$

Since $\hat{m}$ nodes establish an edge to the new node, the expected number of nodes of in-degree $k=\hat{m}$ is

$$
n_{t} P_{t}(\hat{m})=\left(n_{t-1}-\frac{m \hat{m} \alpha n_{t-1}}{e_{t-1}}-m(1-\alpha)\right) P_{t-1}(\hat{m})+1 .
$$


The proof that the limit exists follows by induction on $k$.

Base case. When $k=\hat{m}$, by using Equation 31, $P_{t}(\hat{m})$ can be expressed using the recurrence

$$
P_{t}(\hat{m})=\frac{1}{n_{t}}\left(n_{t-1}-\frac{m \hat{m} \alpha n_{t-1}}{e_{t-1}}-m(1-\alpha)\right) P_{t-1}(\hat{m})+\frac{1}{n_{t}} .
$$

This is a non-autonomous, first-order difference equation. It can be shown by induction over $t$ that the solution of Equation 32 is given by

$$
P_{t}(\hat{m})=\prod_{i=1}^{t} a_{i} P_{0}(\hat{m})+\sum_{i=1}^{t}\left[\prod_{j=i+1}^{t} a_{i}\right] b_{i}
$$

where $a_{t}=\frac{1}{n_{t}}\left(n_{t-1}-\frac{m \hat{m} \alpha n_{t-1}}{e_{t-1}}-m(1-\alpha)\right)$ and $b_{t}=\frac{1}{n_{t}}$. The first term in Equation 33. can be written in terms of the Gamma functions as

$$
\prod_{i=1}^{t} a_{i} P_{0}(\hat{m})=\frac{\Gamma\left(i-\xi_{1}\right) \Gamma\left(i-\xi_{2}\right) P_{0}(\hat{m})}{\Gamma\left(\frac{e_{0}}{m+\hat{m}}+i\right) \Gamma\left(n_{0}+i\right)}
$$

where $\xi_{1}$ and $\xi_{2}$ are constant real numbers that do not depend on time. Moreover,

$$
\lim _{t \rightarrow \infty} \frac{\Gamma\left(t-\xi_{1}\right) \Gamma\left(t-\xi_{2}\right) P_{0}(\hat{m})}{\Gamma\left(\frac{e_{0}}{m+\hat{m}}+t\right) \Gamma\left(n_{0}+t\right)}=0 .
$$

It can further be shown that the second term in Equation 33 is the convergent series

$$
\sum_{i=1}^{\infty}\left[\prod_{j=i+1}^{\infty} a_{i}\right] b_{i}=\frac{m+\hat{m}}{m+\hat{m}+m^{2}+m \hat{m}-m^{2} \alpha} .
$$

Therefore,

$$
\lim _{t \rightarrow \infty} P_{t}(\hat{m})=\frac{m+\hat{m}}{m+\hat{m}+m^{2}+m \hat{m}-m^{2} \alpha} .
$$

Inductive step. Let $k>\hat{m}$ and assume that $\lim _{t \rightarrow \infty} P_{t}(k)$ exists for all $k>\hat{m}$. For a large enough $t, P_{t-1}(k) \sim P_{t}(k)$ and $P_{t-1}(k+1) \sim P_{t}(k+1)$. By Equation 32 and following a line of argument similar to the one in 28, Theorem 1]:

$$
\left(1+\frac{m \alpha(k+1)}{m+\hat{m}}+m(1-\alpha)\right) P_{t}(k+1) \sim\left(\frac{m \alpha k}{m+\hat{m}}+m(1-\alpha)\right) P_{t}(k) .
$$

By inductive hypothesis

$$
\lim _{t \rightarrow \infty} P_{t}(k+1)=\frac{\frac{m \alpha k}{m+\hat{m}}+m(1-\alpha)}{1+\frac{m \alpha(k+1)}{m+\hat{m}}+m(1-\alpha)} \lim _{t \rightarrow \infty} P_{t}(k) .
$$


Therefore, $\lim _{t \rightarrow \infty} P_{t}(k)$ exists for all $k \geq \hat{m}$.

Equation 30 indicates that the expected number of nodes of in-degree $k \geq \hat{m}$ is equal to the difference between the expected number of nodes of in-degree $k$ selected at time $t-1$ by the attachment process and the expected number of nodes of in-degree $k-1$ that establish an edge with the new node.

Corollary 2 characterizes the in-degree distribution of the network.

Corollary 2. If $k \geq \hat{m}$, then the asymptotic behavior of the expected complementary cumulative in-degree distribution satisfies

$$
\bar{F}_{\infty}(k)= \begin{cases}\left(\frac{m}{1+m}\right)^{k-\hat{m}} & , \alpha=0 \\ \frac{\Gamma(\hat{m}+m \hat{m}+m) \Gamma(k)}{\Gamma(\hat{m}) \Gamma\left(k+\frac{m+\hat{m}}{m}\right)} & , \alpha=1 \wedge \hat{m} \geq 1 \\ \frac{\Gamma\left(\frac{\hat{m}+m(1+m+\hat{m}-\alpha m)}{m \alpha}\right) \Gamma\left(k+\frac{(m+\hat{m})(1-\alpha)}{\alpha}\right)}{\Gamma\left(\frac{\hat{m}+m(1-\alpha)}{\alpha}\right) \Gamma\left(\frac{\hat{m}+m(m+\hat{m}+k \alpha+(m+\hat{m}) \alpha+1)}{m \alpha}\right)} & , 0<\alpha<1\end{cases}
$$

Proof. Let $P_{\infty}(k)$ denote the limit of $P_{t}(k)$ as $t$ tends to infinity. According to Theorem 2. $P_{\infty}(k)$ can be written as

$$
P_{\infty}(k)= \begin{cases}\frac{m+\hat{m}}{m^{2}+m \hat{m}+m+\hat{m}-\alpha m^{2}} & , k=\hat{m} \\ \frac{\alpha\left(k m-m^{2}-m \hat{m}-m\right)+m^{2}+m \hat{m}}{\alpha\left(k m-m^{2}-m \hat{m}\right)+m^{2}+m \hat{m}+m+\hat{m}} P_{\infty}(k-1), & k>\hat{m}\end{cases}
$$

Equation 34 defines a recurrence relation that varies as a function of the value of $\alpha$. For $\alpha=0$, the solution of the recurrence is

$$
P_{\infty}(k)=\frac{1}{m+1}\left(\frac{m}{m+1}\right)^{k-\hat{m}} .
$$

For $\alpha=1$ and $\hat{m} \geq 1$, the solution is given by

$$
P_{\infty}(k)=\frac{(m+\hat{m}) \Gamma(\hat{m}+m \hat{m}+m) \Gamma(k)}{m \Gamma(\hat{m}) \Gamma\left(k+\frac{\hat{m}+2 m}{m}\right)} .
$$

Furthermore, for $0<\alpha<1$, the recurrence has solution

$$
P_{\infty}(k)=\frac{(m+\hat{m}) \Gamma\left(\frac{r+m(1+m+\hat{m}-m \alpha)}{m \alpha}\right) \Gamma\left(k+\frac{(m+\hat{m})(1-\alpha)}{\alpha}\right)}{m \alpha \Gamma\left(\frac{m+\hat{m}-m \alpha}{\alpha}\right) \Gamma\left(\frac{\hat{m}+m(m+\hat{m}+k \alpha-(m+\hat{m}) \alpha+\alpha+1)}{m \alpha}\right)} .
$$

Since $\bar{F}_{\infty}(k)=\mathbb{P}[K \geq k]=1-\sum_{j=\hat{m}}^{k-1} P_{\infty}(j)$, by using Equation 35 Equation 36 and Equation 37, the desired result is obtained. 
Consider the plots in Figure 5 They summarize experiments performed on three sequences of networks generated by the algorithm in Definition 1 from the complete graph with 3 nodes. In the three sequences the parameters $m=5$ and $\hat{m}=3$ are fixed. However, each sequence uses a different attachment parameter: $\alpha=0.0, \alpha=0.6$, and $\alpha=1.0$, respectively. The plots in Figure 5 summarize the degree distribution and the complementary cumulative degree distribution for each one of the three sequences of networks. The main observation is that the simulated distributions approach the theoretical limits, a result that follows from Theorem 2 and Corollary 2.

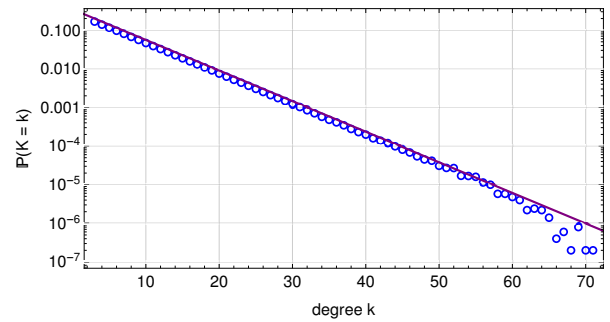

(a)

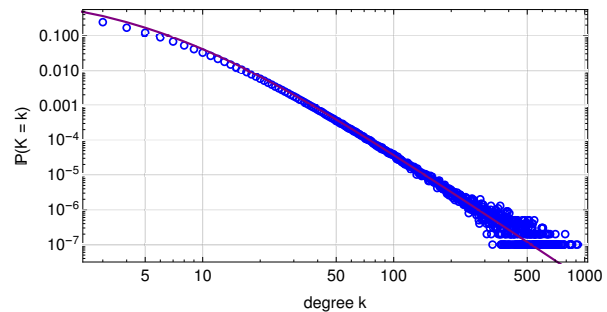

(c)

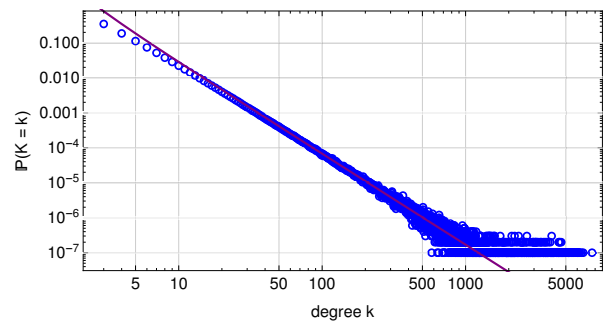

(e)

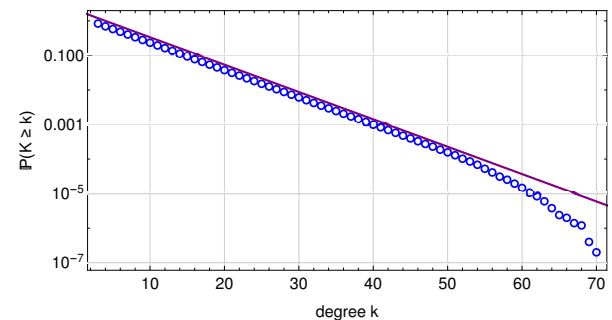

(b)

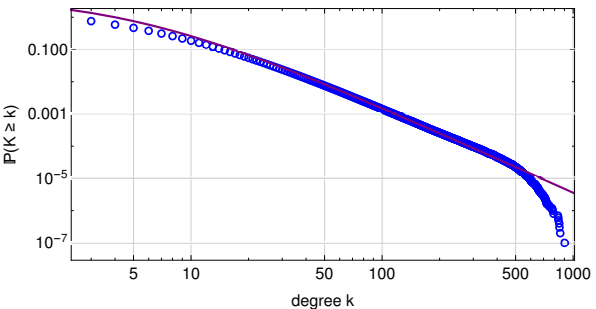

(d)

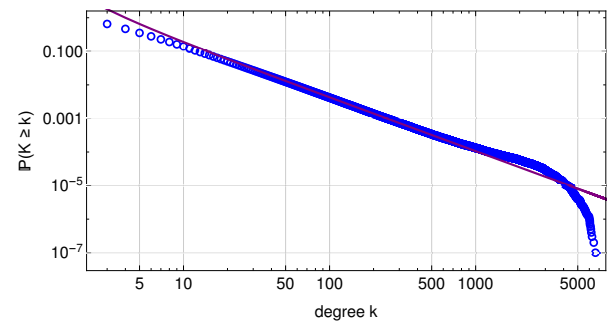

(f)

Fig. 5: Degree distributions and complementary cumulative degree distributions for three sequences of networks. The solid lines represent the average of the ccdf of 100 runs of the model; dashed lines represent the predictions for $m=5, \hat{m}=3$ and $\alpha=0.0$ in (a) and (b); $\alpha=0.6$ in (c) and (d); $\alpha=1.0$ in (e) and (f). 


\section{$6 \quad$ Results}

This section showcases an application of Theorem 1 and the EM Algorithm (i.e., Algorithm 1). It validates the proposed approach to estimate the contribution of the attachment mechanisms in an empirical citation network.

The High Energy Physics Theory (HEP-Th) citation network, a publicly available dataset compiled by ArXiv for the KDD Cup 2003 competition [21, is used. This dataset covers a network of 27770 papers and 352807 citations among them. Each paper has a unique identifier and is annotated with a time-stamp corresponding to its publication date.

The seed network $G_{0}=\left(V_{0}, E_{0}\right)$ has as nodes $V_{0}$ the papers published in February 1992 and the papers cited by them. There is a directed edge $(u, v) \in E_{0}$ from paper $u$ to paper $v$ if and only if paper $u$ cites paper $v$, for any $u, v \in V_{0}$. In total, the seed network $G_{0}$ has 4 nodes and 2 directed edges. The sequence $\left(G_{t}\right)_{t \leq 24284}$ is built by sorting the papers in the HEP-Th network not present in $V_{0}$ in ascending order by time-stamps. That is, the first published paper after February 1992 comes first and identifies the time $t=1$, the second one identifies the time $t=2$, and so on. There is a total of $T=24284$ papers considered in this sequence. Starting from the seed network $G_{0}$, at each time step $t>0$, the network $G_{t}=\left(V_{t}, E_{t}\right)$ is constructed as follows:

- the set of nodes $V_{t}$ contains all nodes in $V_{t-1}$, and has a new node $u_{t}$ representing the paper added at time $t$ and a new node for each paper not present in $V_{t-1}$ that is cited by $u_{t}$; and

- the set of edges $E_{t}$ contains all edges in $E_{t-1}$ and adds a new edge $\left(u_{t}, v\right)$ for each paper $v \in V_{t}$ cited by $u_{t}$.

This process results in the sequence $\left(G_{t}\right)_{t \leq 24284}$ of networks, with $\left|V_{24284}\right|=$ 27770 and $\left|E_{24284}\right|=352807$, representing the growth process of the HEP-Th citation network from February 1992 to April 2003.

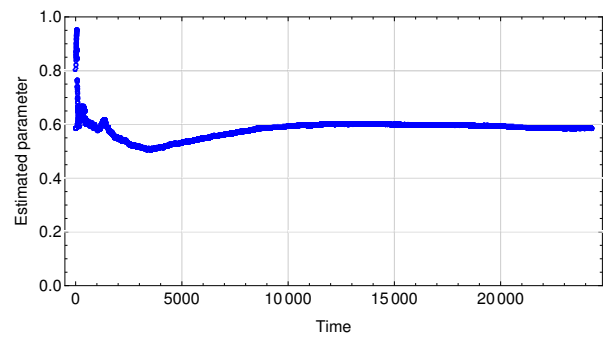

(a)

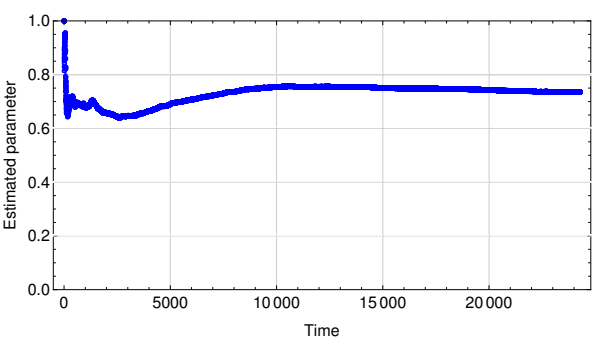

(b)

Fig. 6: Evolution of the estimated parameter for the HepTh network: (a) applying Theorem 1 and (b) applying Algortihm 1 . 
The incidence proportion of the preferential attachment mechanism is estimated by maximizing the $G_{t}$-likelihood at each time $t$, both by using Theorem 1 and the EM Algorithm (i.e., Algorithm 1). The estimation of the attachment mechanisms for $\left(G_{t}\right)_{t \leq 24284}$ is presented in Figure 6, where dots represent the estimated values for the $G_{t}$-likelihood function at each time $t$. By incrementally using Theorem 1 the estimated parameter is calculated to be $\hat{\alpha}_{1}=0.59$. By incrementally using Algorithm 1, the parameter is calculated to be $\hat{\alpha}_{2}=0.74$. With both methods the estimated value becomes stable around time $t=10000$, despite the fact that this value is different for each method. One conjecture that may explain the difference between $\hat{\alpha}_{1}$ and $\hat{\alpha}_{2}$ is related to the way 0-in-degree nodes are accounted for in each case. On the one hand, 0 -in-degree nodes contribute roots to the $G_{t}$-likelihood function (Definition 3) used in Theorem 1 . On the other hand, the E-step of the EM-algorithm ignores 0-in-degree nodes; thus, these nodes do not ultimately contribute to the average computed by the algorithm. Nevertheless, it is important to note that 0 -in-degree nodes, in general, could contribute useful information for parameter estimation. In the case of the HEP-Th citation network the 0-in-degree nodes identify papers that are not cited. It remains as part of the future work to study extensions of the EM Algorithm that consider 0-in-degree nodes.

Based on the estimated parameters $\hat{\alpha}_{1}$ and $\hat{\alpha}_{2}$, Theorem 2 and Corollary 2 are applied to find the theoretical in-degree distribution. The value of the parameter $m$ is estimated using the empirical degree distribution and the estimated parameters; in this case study, $m$ is found to be 12 . Note that, because of the form the network is built, the parameter $\hat{m}$ is 0 (i.e., the networks do not respond to incoming nodes). Figure 7 illustrates the relationship between the theoretical and empirical complementary cumulative degree distributions on $G_{24284}$.

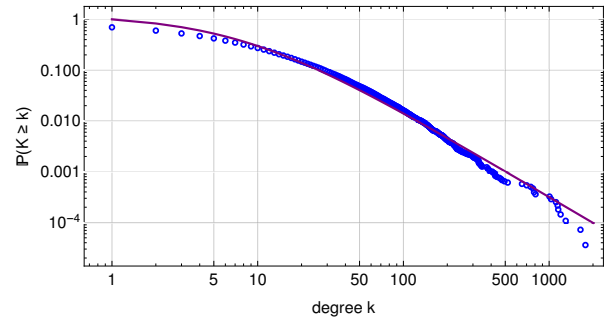

(a)

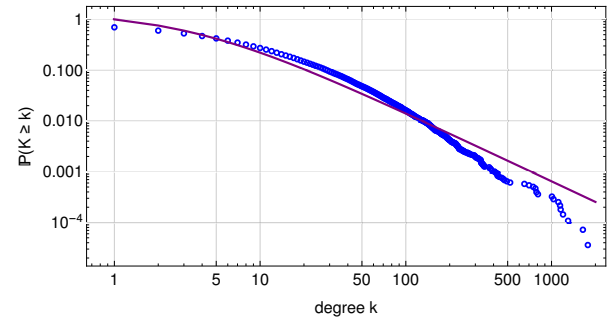

(b)

Fig. 7: Complementary cumulative degree distribution for the HEP-Th network. Solid lines represent the predictions and dots represent the actual values for the empirical complementary degree distributions. (a) $m=12, \hat{m}=0$, and $\hat{\alpha}_{1}=0.59 ;$ (b) $m=12, \hat{m}=0$, and $\hat{\alpha}_{2}=0.74$.

The estimated values have a better fit when the attachment estimate is $\hat{\alpha}_{1}=$ 0.59. In this case, the fit can serve as a witness of the fact that mixed attachment 
models can be used to recreate the behavior experimental networks. The case of the complementary cumulative degree distribution when using the estimate $\hat{\alpha}_{2}=0.74$ may suggest that the actual contribution of the 0 -in-degree nodes, ignored by the EM Algorithm, weights negatively against the estimation process.

\section{Conclusion and Future Work}

Preferential attachment models explain the formation of power-laws in the tail of degree distribution. Such models capture the evolution of the number of connections of a small - yet significant - number of nodes with extremely large degrees. However, preferential attachment alone falls short in describing the behavior of the large majority of nodes with smaller degrees.

To overcome this limitation, mixed attachment models contemplate how degree distributions may result from a combination of multiple mechanisms. Our work is novel for it presented conditions guaranteeing that the prevalence estimate of preferential and random attachment mechanisms represents a local maximum of the likelihood function. We used the expectation maximization algorithm to find the maximum-likelihood estimate of the contribution of the two mechanisms. Our results showed that if the algorithm is applied without satisfying the proposed conditions, then the estimate fails to converge to a stationary value.

Finally, we applied the proposed approach to estimate the prevalence of random and preferential attachment mechanism in citation networks of academic papers. The estimate is evaluated by comparing the empirical degree distribution to the theoretical distribution evaluated at the estimated parameter. The results showed that mixed attachment models are able to recreate the behavior of nodes with both small and large degrees.

Future work on extending the proposed model to include new attachment and response mechanisms that can update edges and even generate clustering should be pursued. For instance, considering link creation rates based on out-degree distributions (e.g., as proposed in [20]) and internal rewiring (e.g., as proposed in [18), should be considered. Furthermore, the analysis of the likelihood functions, and the in- and out-degree distributions of the extended models should also be investigated. New applications to other empirical networks should also be considered, taking into account the rich experience already available with mixed attachment networks. Finally, extensions of the EM Algorithm that take into account 0-in-degree nodes must be studied in order to apply these techniques to, e.g., tree-like empirical networks.

Acknowledgments. Thanks are due to D. Ruiz and F. Amaya for valuable feedback and fruitful discussions. The three authors were in part supported by the Ministry of Information Technologies and Telecommunications of Colombia (MinTIC), and the Colombian Administrative Department of Science, Technology and Innovation (COLCIENCIAS) through the Center of Excellence and Appropriation in Big Data and Data Analytics (CAOBA). 


\section{References}

1. R. Albert and A.-L. Barabási. Statistical mechanics of complex networks. Reviews of Modern Physics, 74(1):47-97, 2002.

2. L. A. N. Amaral, A. Scala, M. Barthelemy, and H. E. Stanley. Classes of small-world networks. Proceedings of the National Academy of Sciences, 97(21):11149-11152, 2000.

3. T. M. Apostol. Mathematical analysis. Addison Wesley Publishing Company, 1974.

4. A. L. Barabási and R. Albert. Emergence of scaling in random networks. Science, 286:509-512, 1999.

5. G. Bianconi and A.-L. Barabási. Competition and multiscaling in evolving networks. Europhysics Letters (EPL), 54(4):436-442, 2001.

6. L. Bordes, D. Chauveau, and P. Vandekerkhove. An em algorithm for a semiparametric mixture model. Computational Statistics and Data Analysis, 51:5429-5443, 2007.

7. A. D. Broido and A. Clauset. Scale-free networks are rare. CoRR, abs/1801.03400, 2018.

8. D. S. Callaway, J. E. Hopcroft, J. M. Kleinberg, M. E. J. Newman, and S. H. Strogatz. Are randomly grown graphs really random? Physical Review E, 64(4):041902, 2001.

9. A. Clauset, C. R. Shalizi, and M. E. J. Newman. Power-law distributions in empirical data. SIAM Review, 51(4):661-703, 2009.

10. A. P. Dempster, N. M. Laird, and D. B. Rubin. Maximum likelihood from incomplete data via the EM algorithm. Journal of the Royal Statistical Society. Series $B$ (Methodological), 39(1):1-38, 1977.

11. J. Diebolt and G. Celeux. Asymptotic properties of a stochastic EM algorithm for estimating mixing proportions. Communications in Statistics. Stochastic Models, 9(4):599-613, 1993.

12. S. N. Dorogovtsev and J. F. F. Mendes. Evolution of networks with aging of sites. Physical Review E, 62(2):1842-1845, 2000.

13. S. N. Dorogovtsev, J. F. F. Mendes, and A. N. Samukhin. Structure of growing networks with preferential linking. Physical Review Letters, 85(21):4633-4636, 2000 .

14. L. Giot, J. S. Bader, C. Brouwer, A. Chaudhuri, B. Kuang, Y. Li, Y. Hao, C. Ooi, B. Godwin, E. Vitols, et al. A protein interaction map of Drosophila melanogaster. Science, 302(5651):1727-1736, 2003.

15. K. Ikehara and A. Clauset. Characterizing the structural diversity of complex networks across domains. CoRR, abs/1710.11304, 2017.

16. M. O. Jackson and B. W. Rogers. Meeting strangers and friends of friends: How random are social networks? American Economic Review, 97(3):890-915, 2007.

17. D. Ke and T. Yi. Growing networks based on the mechanism of addition and deletion. Chinese Physics Letters, 21(9):1858-1860, 2004.

18. P. L. Krapivsky and S. Redner. Organization of growing random networks. Phys. Rev. E, 63:066123, May 2001.

19. P. L. Krapivsky, S. Redner, and F. Leyvraz. Connectivity of growing random networks. Physical Review Letters, 85(21):4629-4632, 2000.

20. P. L. Krapivsky, G. J. Rodgers, and S. Redner. Degree distributions of growing networks. Phys. Rev. Lett., 86:5401-5404, Jun 2001.

21. J. Leskovec, J. Kleinberg, and C. Faloutsos. Graph evolution: Densification and shrinking diameters. ACM Trans. Knowledge Discovery from Data, 1(1):1-40, 2007. 
22. S. Li, C. M. Armstrong, N. Bertin, H. Ge, S. Milstein, M. Boxem, P.-O. Vidalain, J.-D. J. Han, A. Chesneau, T. Hao, et al. A map of the interactome network of the Metazoan C. elegans. Science, 303(5657):540-543, 2004.

23. Z. Liu, Y.-C. Lai, N. Ye, and P. Dasgupta. Connectivity distribution and attack tolerance of general networks with both preferential and random attachments. Physics Letters A, 303(5-6):337-344, 2002.

24. G. McLachlan and T. Krishnan. The EM Algorithm and Extensions: Second Edition. John Wiley \& Sons, 2007.

25. M. Newman. The structure and function of complex networks. SIAM Review, 45(2):167-256, 2003.

26. M. E. Newman. Modularity and community structure in networks. Proceedings of the National Academy of Sciences, 103(23):8577-8582, 2006.

27. S. Redner. How popular is your paper? An empirical study of the citation distribution. The European Physical Journal B-Condensed Matter and Complex Systems, 4(2):131-134, 1998.

28. D. Ruiz and J. Finke. Stability of the Jackson-Rogers model. In 2017 IEEE 56th Annual Conference on Decision and Control (CDC), pages 1803-1808. IEEE, 2017.

29. A. Said, E. De Luca, and S. Albayrak. How social relationships affect user similarities. In Proceeding of the 2010 Workshop on Social Recommender Systems, pages $1-4,2010$.

30. Z.-G. Shao, X.-W. Zou, Z.-J. Tan, and Z.-Z. Jin. Growing networks with mixed attachment mechanisms. Journal of Physics A: Mathematical and General, 39(9):2035-2042, 2006.

31. P. Sheridan, Y. Yagahara, and H. Shimodaira. A preferential attachment model with Poisson growth for scale-free networks. Annals of the Institute of Statistical Mathematics, 60(4):747-761, 2008.

32. S. H. Strogatz. Exploring complex networks. Nature, 410(6825):268-276, Mar. 2001.

33. G. Szabó, M. Alava, and J. Kertész. Clustering in complex networks. In Complex Networks, pages 139-162. Springer, 2004.

34. B. Tadić. Dynamics of directed graphs: the world-wide web. Physica A: Statistical Mechanics and its Applications, 293(1):273 - 284, 2001.

35. W. Wang, Q. Zhang, and T. Zhou. Evaluating network models: A likelihood analysis. EPL (Europhysics Letters), 98(2):28004, 2012.

36. Q. Zhang, X. Xu, Y. Zhu, and T. Zhou. Measuring multiple evolution mechanisms of complex networks. Scientific Reports, 5(1):10350, 2015. 